\title{
The Use of the Three-Pedicle Dermal Flap in Preventing Mastectomy for Large and/or Ptotic Breasts: A Novel Concept to prevent NAC survival in Two Stages Reconstruction
}

Koulermou $\mathbf{G}^{*}$

Department of Plastic Surgery and Burn Unit, Nicosia General Hospital, Nicosia, Cyprus

*Corresponding author: Koulermou G, Department and Burn Unit Nicosia General Hospital, Nicosia, Cyprus, Tel: +35722603000; E-mail: gkoulerm@spidernet.com.cy Received date: Nov 22, 2016; Accepted date: Jan 03, 2017; Published date: Jan 10, 2017

Copyright: (c) 2017 Koulermou G. This is an open-access article distributed under the terms of the Creative Commons Attribution License, which permits unrestricted use, distribution, and reproduction in any medium, provided the original author and source are credited.

\begin{abstract}
Preventing mastectomy (or prophylactic or risk reducing mastectomy) has been a major area of interest within the field of surgical methods during the last years, as a result of the recognition that the genetic basis of patients with BRCA 1 and 2 mutations, and those with very strong family history, acts as an indicative factor. Whereby, the procedure appears to be a cancer disease preventing method at very high risk patients.

Firstly, surgical management of Preventing Mastectomy and Implant Based Reconstruction is easier in small and moderate ptotic breasts, rather than in big and extensive breasts. Whereas, in patients with macromastia a reduction or mastopexy procedure is needed in order to achieve the desirable shape and position of the breast. In this category of patients viability and survival of Nipple Areola Complex is unsecure. Thus, this technique describes the performance of Preventing Mastectomy using a three pedicle dermal flap that supports blood supply of the NAC and increases the survival rate. All patients who underwent the three pedicle technique had been operated at the Plastic Surgery Department of Nicosia General Hospital between October of 2013 and October of 2015, by the writer.
\end{abstract}

Keywords: Preventing mastectomy; Risk reducing mastectomy; NAC sparing mastectomy; Dermal flap

\section{Introduction}

Preventing Mastectomy (or risk reducing mastectomy) is performed in order to reduce the risk of breast cancer in high risk patients, in patients with BRCA mutation, and those with very strong family history. This is achieved by removing mammary gland based on the oncological principles, and by reducing skin by a safety way with preservation of the nipple.

Target of the procedure is to remove the mammary gland with a NAC preservation. The Skin Reduction pattern is often needed to minimize the skin excess and give the breast an aesthetically good result. In fact, NAC sparing mastectomy techniques have improved our reconstructive options and aesthetic outcomes, giving a solution to the increasing expectations of a good aesthetic result and oncological safety $[1,2]$.

It is widely accepted that women with large and ptotic breasts, where a reduction pattern during the mastectomy is required, appear to have high rates of skin flaps and NAC complications. [3,4] Depending on the different level of ptosis of the breast, different approaches are needed to ensure NAC and skin flaps viability [5].

The use of the three-pedicle flap a modification of the classical vertical bipedicle flap creates a more viable solution for NAC survival in large and/or ptotic breasts.

\section{Surgical Technique}

The patient is marked in standing position (the same procedure we follow for a reduction mammoplasty using wise pattern, although with some modifications in measurements) accordingly to the patients breast size (Figure 1) [2].

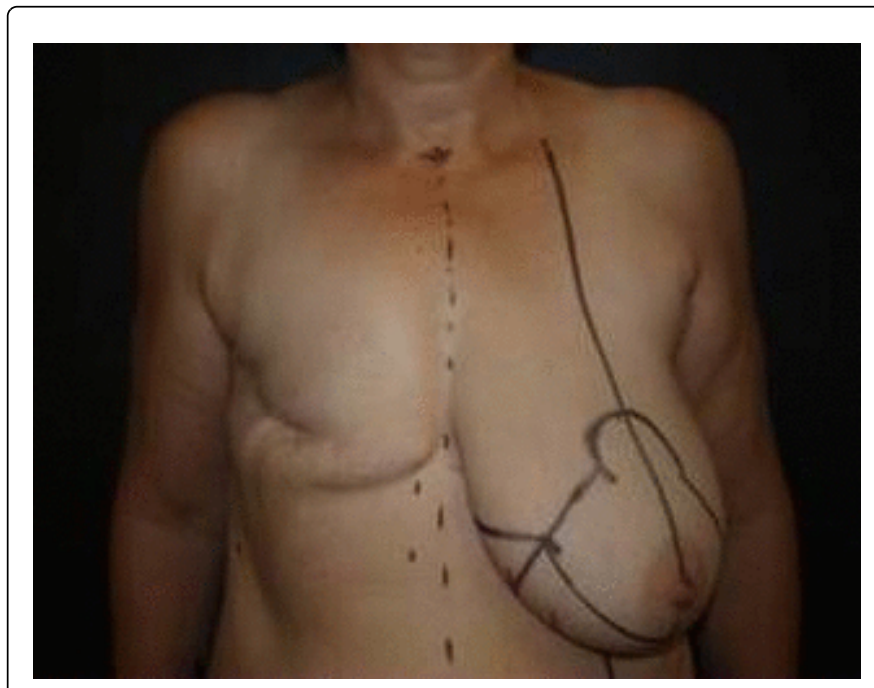

Figure 1: Preoperative marking of wise pattern-three pedicle pattern.

The first step is the deepithelization of the three-pedicle flaps, which also separates them from the lateral part of the breast. The three dimensions of the blood supply flap are: inferiorly, superiorly and 
Citation: Koulermou GM (2017) The Use of the Three-Pedicle Dermal Flap in Preventing Mastectomy for Large and/or Ptotic Breasts: A Novel Concept to prevent NAC survival in Two Stages Reconstruction. Arch Surg Oncol 3: 117. doi:10.4172/2471-2671.1000117

Page 2 of 4

laterally based. These will provide a better vascularization to the NAC by preserving vascularity coming from branches of the internal mammary and lateral thoracic arteries, which in turn run in the subcutaneous tissue and communicate with each other (Figure 2). The mastectomy is then performed by dissecting both, superiorly and inferiorly, in the typical plane between the subcutaneous fat and breast parenchyma.

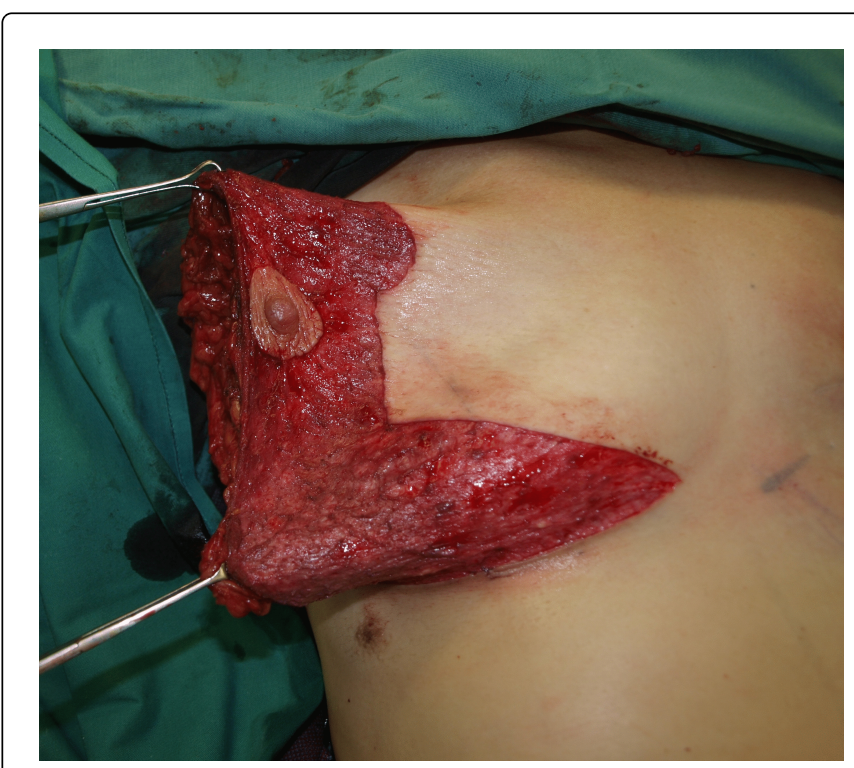

Figure 2: The three pedicle dermal flap.

Following this, a submuscular pocket is perform under the pectoralis major, until it is attached to the rectus abdominus fascia and serratus anterior. Elevation of the pectoralis muscle will provide a complete coverage to the expander and a good width for the mastectomy skin flaps.

The underlying dermal flap increases blood supply to the skin pillars and T-point, decreasing complication rates 6 of skin flaps and NAC necrosis. Inflation of the expander begins at the third postoperative week.

Gradually inflation of the expander minimizes the tension on the NAC and skin flaps a second parameter that decreases the complication rates. A second stage follows the maturity of the capsule, where the replacement of the expander with the definitive implant takes place. The surgical procedure is performed through the sub mammary incision. During the second stage minor corrections of the shape can take place by a more safe way than the time of the mastectomy $[1,6]$.

\section{Discussion}

Preventing Mastectomy in women with large and ptotic breasts, combines a Nipple preserving and a reduction mastectomy technique. The determinant factors for the achievement of a good esthetic result are: the accurate preoperative marking, and the safety surgical technique to provide NAC's and skin flaps viability.

In order to ensure satisfactory cosmetic results, these patients frequently require extensive skin reduction and removal of nipple position [2,7-9]. A causal pattern is one of the techniques chosen to facilitate large and/or ptotic breasts. Besides, it allows for greater control over the final shape of the reconstruction performing reduction or mastopexy at the same time [5]. Although this can result in favorable shape and symmetry, it has a high rate of NAC and skin pillars complications,such as superficial epidermolysis, or full thickness necrosis of the NAC, wound dehiscence, and implant exposure [10,11].

Incidence of skin necrosis, especially at the T-junction, range from 20 to 30 percent by some writers $[4,6,12,13]$.

NAC in large and/or ptotic breasts have insufficient blood supply due to the long distance from stern al notch ,or inframammary fold .

The additional blood supply from the three pedicle dermal flap provides a third dimension blood supply to the flap by preserving vascularity coming from branches of the internal mammay and lateral thoracic arteries which run in the subcutaneous tissue and communicate with each other [2].

Immediate reconstruction with implants may sometimes result to NAC or skin flaps necrosis due to the tension on the flaps. The two stages reconstruction with the use of tissue expanders could minimize the tension with gradually inflation of the expander $[6,10,13]$.

The advantage of using the expander in this method is the fact that it allows for a larger expansion, a more natural ptotic shape and applies less pressure on NAC and the skin flaps of mastectomy [14,15]. Therefore facilitating the tissues with a better blood supply level, hence minimizing the necrosis incidence.

Key features of the technique that uniquely mark it compared to others are the following: a) The use of a three pedicle dermal flap which will provide additional blood supply; b) The use of the tissue expander that will minimize the tension on NAC. These factors ensure a good and safe solution to NAC preservation in addition to viability, by giving a good aesthetic result (Figure 3 ).

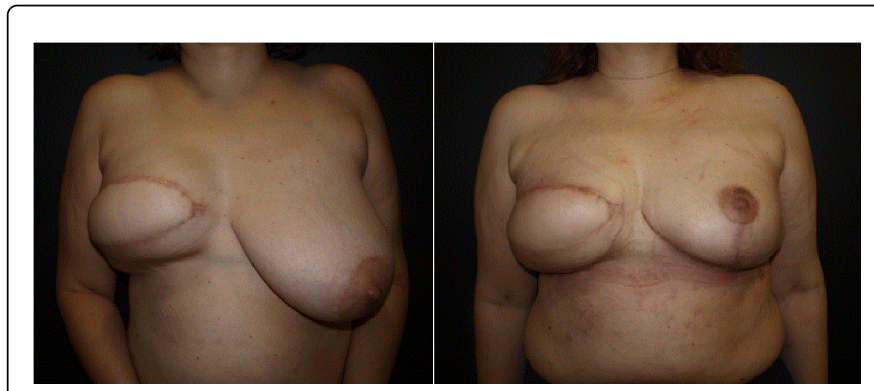

Figure 3: Preoperative and postoperative appearance of the patient with preventing mastectomy on left breast, using the technique of the three pedicle flap. On the right side breast reconstruction after mastectomy with Latissimus dorsi flap.

The three pedicle dermal flap described for the first time, give the chance to achieve a good and safe aesthetic result in women with large and ptotic breasts who undergo a preventing mastectomy (Figures $4-7)$. 
Citation: Koulermou GM (2017) The Use of the Three-Pedicle Dermal Flap in Preventing Mastectomy for Large and/or Ptotic Breasts: A Novel Concept to prevent NAC survival in Two Stages Reconstruction. Arch Surg Oncol 3: 117. doi:10.4172/2471-2671.1000117

Page 3 of 4

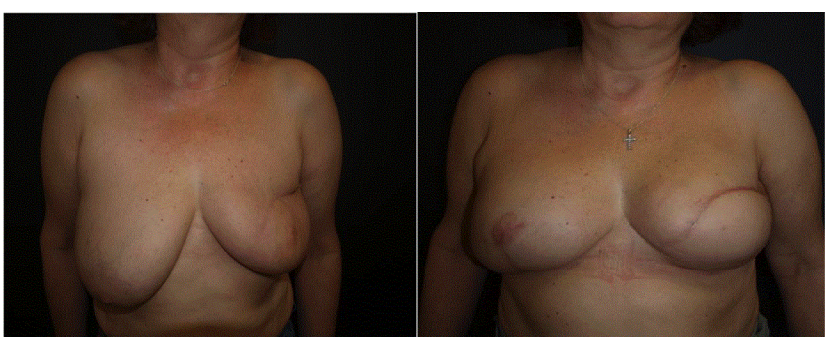

Figure 4: Preoperative and postoperative appearance of the patient with preventing mastectomy on right breast, using the technique of the three pedicle flap. On the left side breast reconstruction after mastectomy with Latissimus dorsi flap.

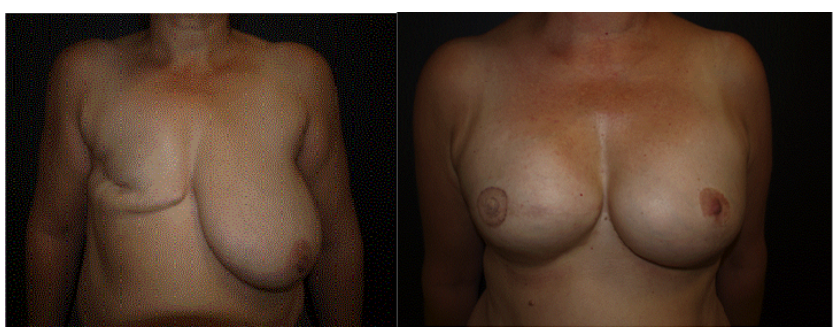

Figure 5: Preoperative and postoperative appearance of the patient with preventing mastectomy on left breast, using the technique of the three pedicle flap. On the right side a two stages breast reconstruction after mastectomy with tissue expander and implant.

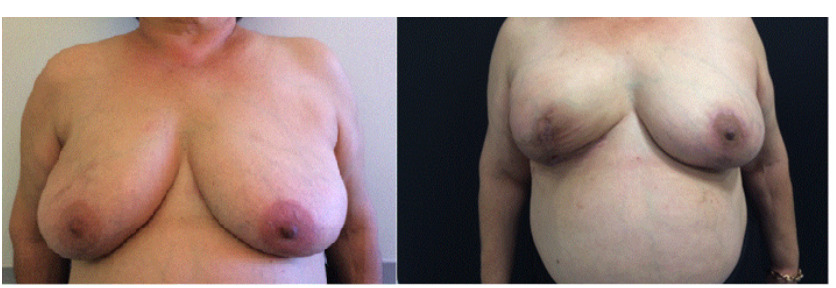

Figure 6: Preoperative image, and postoperative appearance of the patient with preventing mastectomy on left breast, using the technique of the three pedicle flap. On the right side breast mastectomy with an one stage implant based reconstruction. You can see a small wound dehiscence at the triple point.

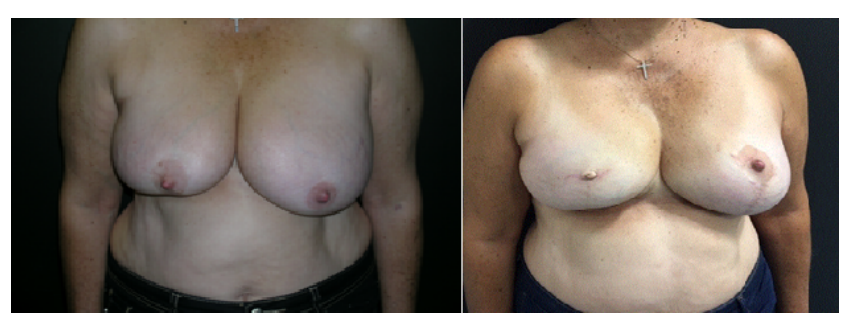

Figure 7: Preoperative image, and postoperative appearance of the patient with preventing mastectomy on left breast, using the technique of three pedicle flap. On the right side a mastectomy with a two stages reconstruction with tissue expander and implant.

Between the 42 patients who underwent a three pedicle preventing mastectomy the last two years (from October 2013 to October 2016), Skin Flaps viability problems appear in 6 patients (14.28\%) 4 patients with Superficial necrosis of the nipple $(9.52 \%)$, one patient with Epidermolysis (2.38\%), and one patient with full thickness necrosis as part of the T-junction (2.38\%).

\section{Conclusion}

The described innovation with the use of the three pedicle dermal flap, a modification of the well known bipedicle flap, gives the chance of a better blood supply to the nipple support and an increase rate on NAC survival and the skin pillars as well. The two stages reconstruction with the use of a tissue expander and the gradually inflation, decreases the possibility of a NAC necrosis due to tension on the Nipple Areola Complex.

This is the first time that a three-pedicle dermal flap has been used in preventing mastectomy for large and/or ptotic breasts. Therefore it plays a pivotal role in the field of preventing mastectomy techniques as it minimizes the complications that usually appear when a NAC transposition at a new place is needed due to breast ptosis and skin excess exist.

\section{References}

1. Verheyden CN (1998) Nipple-sparing total mastectomy of large breasts: The role of tissue expansion. Plast Reconstr Surg 101: 1494-1500.

2. Skoll PJ, Hudson DA (2002) Skin-sparing mastectomy using a modified Wise pattern. Plast Reconstr Surg 110: 214-217.

3. Rusby J, Gui G (2010) Nipple sparing mastectomy in women with large or ptotic breasts. Plast Reconstr Aesthetic Surg 63: e754-e755.

4. Dietz J, Lundgren P, Veeramani A, Rourke CO, Bernard S, et al. (2012) Autologous Inferior Dermal Sling (Autoderm) with Concomitant SkinEnvelope Reduction Mastectomy: An Excellent Surgical Choice for women with Macromastia and Clinically Significant Ptosis. Ann Surg Oncol 19: 3282-3288.

5. Salgarello M, Visconti G, Barone-Adesi L, Franceschini G, Magno S, et al. (2012) Inverted-T Skin-Reducing mastectomy with Immediate Implant Reconstruction Using the Submuscular-Subfascial Pocket. Plast Reconstr Surg 130:31-41.

6. Losken A, Collins B, Carlson G (2010) Dual-plane prosthetic reconstrutionusing a modified Wise pattern mastectomy and fasciocutaneous flap in women with macromastia. Plas Reconstr Surg 126: 731-738. 
Citation: Koulermou GM (2017) The Use of the Three-Pedicle Dermal Flap in Preventing Mastectomy for Large and/or Ptotic Breasts: A Novel Concept to prevent NAC survival in Two Stages Reconstruction. Arch Surg Oncol 3: 117. doi:10.4172/2471-2671.1000117

Page 4 of 4

7. Veronesi P, Ballardini B, De Lorenzi F, Magnoni F, Lissidini G, et al. (2011) Immediate breast reconstruction after mastectomy. Breast 3: s104s107.

8. Hammond DC, Capraro PA, Ozolins EB, Arnold JF (2002) Use of a skinsparing reduction pattern to create a combination skin-muscle flap pocket in immediate breast reconstruction. Plast Reconstr Surg 110: 206-211.

9. Losken A, Collins BA, Carlson GW (2010) Dual plane prosthetic reconstruction using the modified Wise pattern mastectomyand fasciocutaneous flap in women with macromastia. Plast Reconstr Surg 126: 731-738

10. Nava M, Maurizio B, Cortinovis U, Ottolenghi J, Riggio E, et al. (2006) Skin- Reducing Mastectomy. Plast Reconstr Surg 118: 603-610.
11. Maurizio BN, Catanuto G, Pennati A, Garganese G, Spano A, et al. (2009) Conservative mastectomies. Aesth Plast Surg 33: 681-686.

12. Carlson GW (2006) Skin Reducing mastectomy. Plast Reconstr Surg 118: 611-613.

13. Rusby JE, Smith BL, Gui GPH (2010) Nipple-sparing mastectomy. BJS 97: 305-316.

14. Hudson PA, Skoll OJ (2002) Complete one stage immediate breast reconstruction with prosthetic material in patients with large or ptotic breasts. Plast Reconstr Surg 110: 487-493.

15. Carlson GW, Bostwick J III, Styblo TM, Moore B, Bried JT, et al. (1977) Skin-sparing mastectomy: Oncologic and reconstruction considerations. Ann Surg 225: 570-575. 